Article

\title{
Efficient Modulation Scheme for Intermediate Relay-Aided IoT Networks
}

\author{
Waleed Shahjehan ${ }^{1,+}$, Shahid Bashir ${ }^{2,+}$, Saleem Latteef Mohammed ${ }^{3,+}$, Ahmed Bashar Fakhri ${ }^{3,+}$, \\ Adeniyi Adebayo Isaiah ${ }^{4,+}$ and Imran Khan ${ }^{1,+}$ and Peerapong Uthansakul ${ }^{5, *,+}$ (D) \\ 1 Department of Electrical Engineering, University of Engineering and Technology Peshawar, \\ Peshawar P.O.B. 814, KPK, Pakistan; waleedshahjehan@gmail.com (W.S.); ikn.eup121@gmail.com (I.K.) \\ 2 Business Studies Department, Namal Institute, 30 km Talagang Road, Mianwali 42250, Pakistan; \\ shahid.bashir@namal.edu.pk \\ 3 Department of Medical Instrumentation Techniques Engineering, Electrical Engineering Technical College, \\ Middle Technical University, Baghdad 10013, Iraq; saleem_lateef_mohammed@mtu.edu.iq (S.L.M.); \\ ahmed_bashar@mtu.edu.iq (A.B.F.) \\ 4 International Institute of Tropical Agriculture (IITA), PBM 98, 109101, Ibadan 200001, Nigeria; \\ aadebayoisaiah@gmail.com \\ 5 School of Telecommunication Engineering, Suranaree University of Technology, \\ Nakhon Ratchasima 30000, Thailand \\ * Correspondence: uthansakul@sut.ac.th \\ + These authors contributed equally to this work.
}

Received: 1 March 2020; Accepted: 18 March 2020; Published: 20 March 2020

\begin{abstract}
With the surge of ubiquitous demand for high-complexity and quality mobile Internet-of-things (IoT) services, new cooperative relaying paradigms have emerged. Motivated by the long and unpredictable end-to-end communication in relay-aided IoT networks, there is a need to introduce novel modulation schemes for very low bit error rate (BER) communications. In this paper, a practical modulation mapping scheme has been proposed to reduce decoding errors. Specifically, a hybrid automatic repeat request (HARQ) system has been used with an intermediate relay to transfer a message from a source to a destination. The design of modulation mapping has been optimized by first formulating the objective as the quadratic assignment problem. Later, the solution to the mapping problem is provided using an iterative search method. To validate the proposed solution, extensive simulations have been performed in MATLAB. The results show that the proposed solution outperforms the conventional relay retransmission and the heuristic design approaches.
\end{abstract}

Keywords: relay-aided; HARQ; IoT network; quadratic assignment

\section{Introduction}

The world is growing at a fast pace, and so is data. The agility and flexibility of big data applications are gradually shaping the Internet-of-things (IoT) [1]. These IoT devices feature unique identities and are accessible from anywhere in the world. The ubiquitous deployment of interconnected devices is expected to grow in the next decade. This exponential growth is broadly supported by the increasing number of mobile devices (e.g., smartphones and tablets), smart sensors serving different markets (e.g., autonomous transportation, industrial controls and wearables), wireless sensors and actuators networks [2-8]. Reliable exchange of data by IoT sensors and actuators is one of the biggest challenges faced when deploying an IoT system. Although this kind of device has evolved radically in the last years, battery life, computation and storage capacity remain limited [9]. This means that they are not suitable for running heavy applications; instead, it is necessary to resort to more powerful computing resources, likely owned by third parties. 
For instance, drones, also known as unmanned aerial vehicles (UAVs), can be employed in many of applications that are difficult or dangerous for humans (e.g., military, disaster or emergency missions) [10-13]. The mode of operation of drones has progressively changed from the traditional remote control by humans to sophisticated autonomous control, able to operate without human intervention. However, as all the processing required to analyze the data and make decisions are performed onboard, flight autonomy decreases substantially. For instance, let us consider an extremely resource-intensive application; say, the search for missing persons in disaster zones using image processing [10]. With regard to the image processing module, as it has no such tight time constraints, it can be forwarded to the relay, which will be responsible for processing the collected data (e.g., video, images or heat sensor data) and transmitting it reliably to the destination nodes. This way, the required decoding errors are tremendously reduced, resulting in an increase in terms of the overall functionality [14].

In order to recover the lost packet for improving reliability at the network layer, one of the most widely used techniques is hybrid automatic repeat request (HARQ) [15]. HARQ in conjunction with relay networks has attracted substantial research interest in recent years [16]. Because data symbols in practical transmission often utilize linear modulations of finite-size constellations (e.g., quadrature phase-shift keying (PSK), or quadrature quadrature amplitude modulation (QAM)), the performances of relay-aided HARQ systems can benefit from modulation diversity, in which each group of $\log _{2} Q$ bits is mapped to a different constellation point across different links and in different transmissions to better exploit channel diversity.

\subsection{Related Works}

There are several known studies on modulation diversity for HARQ [17], relay networks [18,19] and relay-HARQ systems [20]. Despite the promising performance gains, these methods are developed for relay network settings that tend to consume substantial bandwidth for which (re)transmissions on the source to destination link and the relay to destination links occupy orthogonal channels (frequency bands or time slots). In a similar manner, the authors of [21] proposed a HARQ-based minimum mean-square-error equalizer to improve the efficiency of an offset quadrature amplitude modulation-based filter bank sub-carrier. In terms of block error rate, their proposed approach appears to provide significant gains. Leturc et al. in [22] provided an energy-efficient resource allocation scheme for Type-1 HARQ network. With the help of fractional programming and convex optimization framework, they derived the closed-form solution for the resource allocation problem. They also concluded that significant performance gains could be achieved for line-of-sight (LOS) communication between transmitter and receiver. Some other works [23-25] also take into account the physical layer security aspects of HARQ networks.

A novel codeword solution invoking the HARQ mechanism was provided by the authors of [26]. Specifically, they retransmitted the unreliable bits of the message using carefully constructed codewords. Some of the studies have also attempted to improve the throughput of the network. For instance, Saber et al. in [27] utilized the intermediate values of the polar codes to improve upon the effective throughput. An intermediate polar code value was transmitted as redundant bits. The authors of [28] proposed a novel approach to encode the new data bits with the previous transmission. A range of different puncture techniques was also investigated in the literature of HARQ systems. In this regard, the authors of [29] proposed a stopping-tree puncturing method, whereas the authors of [30] provided a quasi-uniform puncturing technique. More recently, the authors of $[31,32]$ exploited the flexible design of the HARQ scheme by proposing the replication of error-prone bits of the data. However, they focused on the traditional long or moderate packet transmission, which may not be more feasible for IoT applications. In this backdrop, the authors of $[33,34]$ provided a novel short packet HARQ design, which, despite degrading the performance, is a more practical approach to handle delay-sensitive applications.

Wang et al. in [35] proposed an index modulation-based multi-hop relaying system. They also derived the outage probability and block error rate expressions. Their proposed sub-carrier activation method improves the performance of orthogonal frequency division multiplex systems. Similar works 
have been reported by the authors of [36-39] wherein the authors provide the interleaving method, deep learning modulation techniques and the random linear network coding methods. The authors of [40] study modulation diversity design for the relay-HARQ configuration in which the source to destination and relay to destination links are co-channel and additive. Such a configuration specifically forms a 2-by-1 multiple-input single-output (MISO) diversity transmission. For this system configuration, we formulate the bit error rate (BER) minimization modulation diversity design in a quadratic assignment problem. As this is NP-hard, our modulation diversity design cannot be solved exactly, even for a moderately sized constellation, such as 16-QAM. Fortunately, there exist a number of heuristic algorithms [41] for its reduced form, known as the quadratic assignment problem, that result in high-quality solutions over the quadratic assignment problem library dataset. The authors of [42] argued that the simultaneous wireless information and power transfer system designed using Gaussian distributed input signals may result in degradation of performance. Thus, they provided a guide for coding-controlled single and multi-user simultaneous wireless information and power transfer systems.

\subsection{Motivation and Contribution}

After careful review of the aforementioned studies, we noted that there is a dearth of efficient modulation schemes for relay-aided IoT networks. This is especially true for the HARQ system, where retransmission occurs frequently for reliable transfer of messages among two devices. In fact, most of the studies have focused on the resource allocation aspect of such networks to improve performance. These schemes do not take into account the retransmission which provides less practical analysis for wireless networks. To partly fill this gap in the literature, this work provides an efficient modulation scheme to reduce the decoding error. In particular, our work adopts an iterated search method that is efficient for solving the quadratic assignment problem formulated from the modulation diversity design problem for minimizing reception BER. The performance of the proposed modulation mapping approach is compared with conventional heuristic and retransmission HARQ methods. It is shown that for a different number of users in the network, the proposed approach outperforms the conventional approaches, even at low power. The results also show the performance improvements and significant BER reduction due to using the proposed approach.

\subsection{Organization}

The remainder of the paper is organized as follows. Section 2 first describes the intermediate relay-aided network model. Section 3 presents the optimal modulation diversity design problem and the proposed solution. Section 4 provides and compares the numerical results to illustrate the benefit of the proposed approach. Finally, Section 5 concludes this work.

\section{System Model}

Similarly to the network setup of $[43,44]$, we consider the relay-aided HARQ network shown in Figure 1, in which there are two transmission phases. In Phase 1, the source node broadcasts its packet to both destination and relay nodes. In Phase 2 of this HARQ setup, upon packet loss notification, both relay and source nodes cooperatively retransmit the lost packet information to the destination. Our goal is to optimize HARQ constellation mappings to improve modulation diversity so as to minimize the packet BER at the receiver.

Denote $\mathcal{C}$ as the primary constellation used by this relay network whose cardinality equals $Q=|\mathcal{C}|$. In the first phase, the source converts a bit sequence of length $\log _{2} Q$ into symbols with Gray mapping $\lambda_{0}:\{0, \ldots, Q-1\} \rightarrow \mathcal{C}$. The bit sequence can be indexed by its decimal equivalence $p \in\{0, \ldots, Q-1\}$. The source transmits $\lambda_{0}[p]$ to the destination via channel $h_{0}$, though $\lambda_{0}[p]$ is simultaneously received by the decode-and-forward (DF) relay. Following the approach of $[20,45]$, we consider that the relay is placed strategically such that it has a negligible decoding error rate. Upon receiving a request for retransmission, the second phase begins with the source and the relay re-mapping $p$ into $\lambda_{1}[p]$ 
and $\lambda_{2}[p]$, respectively. Specifically, we note that in the second phase, the source retransmits the same message again towards the destination. In the meantime, the relay also forwards the same message to the destination. Therefore, the messages from source and relay are remapped in the form of $\lambda_{1}[p]$ and $\lambda_{2}[p]$. In general, we have three distinct mappings $-\lambda_{0}, \lambda_{1}$ and $\lambda_{1}$, such that $\lambda_{1} \neq \lambda_{0}$ and $\lambda_{2} \neq \lambda_{0}$. The remapped symbols are transmitted simultaneously on the same channel band to the destination via channels $h_{1}$ (from source to destination) and $h_{2}$ (from the relay to destination). In summary, the signals received at the destination during the two phases are, respectively,

$$
\begin{aligned}
& y_{1}=h_{0} \lambda_{0}[p]+v_{1} \\
& y_{2}=h_{1} \lambda_{1}[p]+h_{2} \lambda_{2}[p]+v_{2}
\end{aligned}
$$

where $v_{1}, v_{2} \sim \mathcal{C N}\left(0, \sigma_{v}^{2}\right)$ are additive channel noises. Throughout this work, we assume fading wireless channels $h_{0}, h_{1}$ and $h_{2}$ to follow independent Rician distribution.

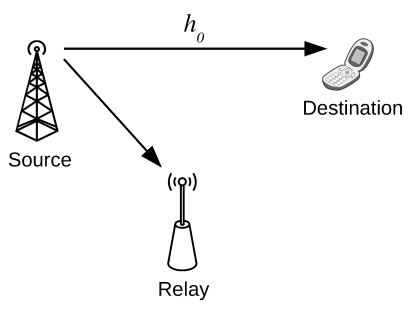

(a) Phase 1

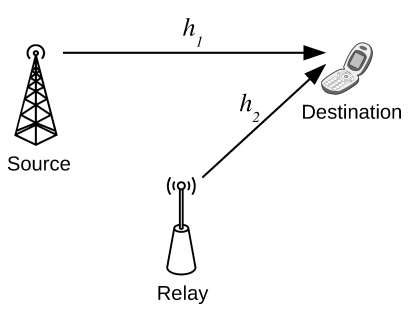

(b) Phase 2

Figure 1. Intermediate relay-aided HARQ network.

Assume that the destination acquires perfect channel state information (CSI). Based on the received symbols $y_{1}$ and $y_{2}$, the destination decodes the data by identifying index $p$ via maximum likelihood (ML) detection:

$$
\min _{\hat{p}}\left|y_{1}-h_{0} \lambda_{0}[\hat{p}]\right|^{2}+\left|y_{2}-h_{1} \lambda_{1}[\hat{p}]-h_{2} \lambda_{2}[\hat{p}]\right|^{2}
$$

\section{Proposed Solution}

In this section, we elaborate on the numerical approach for computing the input cost matrix of the quadratic assignment problem and provide an efficient algorithm to obtain a numerical solution. We also shed light on the conventional heuristic approach for solving the problem.

\subsection{Proposed BER Minimization Solution}

Assume that the information-bearing index $p$ follows a uniform distribution; then, the BER using pairwise error probability (PEP) [46] can be written as

$$
P_{B E R}=\sum_{p=0}^{Q-1} \sum_{q=0}^{Q-1} P_{P E P}(q \mid p) \frac{B[p, q]}{Q},
$$

where $P_{P E P}(q \mid p)$ denotes the probability for the ML decoder to prefer $q$ over $p$ when $p$ is actually transmitted; $B[p, q]$ represents the Hamming distance normalized by $\log _{2} Q$. According to (2), we have

$$
P_{P E P}(q \mid p)=P_{h_{0}, h_{1}, h_{2}, v_{1}, v_{2}}\left\{\vartheta\left(p, q, \lambda_{1}, \lambda_{2}\right)<0\right\} .
$$


in which, given random channels and random noise variables $h_{0}, h_{1}, h_{2}, v_{1}, v_{2}, \vartheta$ is defined as

$$
\begin{aligned}
& \vartheta=\left|h_{0}\left(\lambda_{0}[p]-\lambda_{0}[q]\right)+v_{1}\right|^{2}-\left|v_{1}\right|^{2}+ \\
& \left|h_{1}\left(\lambda_{1}[p]-\lambda_{1}[q]\right)+h_{2}\left(\lambda_{2}[p]-\lambda_{2}[q]\right)+v_{2}\right|^{2}-\left|v_{2}\right|^{2} .
\end{aligned}
$$

In other words, given indices $p, q$ and the remapping scheme $\lambda_{1}, \lambda_{2}$, the pairwise error event, is equivalent to $\vartheta<0$. In order to formulate the quadratic assignment problem, we introduce binary variables

$$
x_{p i j}= \begin{cases}1, & \text { if } \lambda_{1}[p]=\lambda_{0}[i] \text { and } \lambda_{2}[p]=\lambda_{0}[j] \\ 0, & \text { otherwise. }\end{cases}
$$

Let us denote $\mathbf{x}=\left\{x_{p i j} \mid p, i, j=0, \ldots, Q-1\right\}$ and the constraint set as

$$
\mathcal{P}=\left\{\mathbf{x}: \sum_{p=0}^{Q-1} x_{p i j}=1, x_{p i j} \in\{0,1\}\right\} .
$$

We further denote $\mathcal{I}$ and $\mathcal{J}$ as in (6) by replacing the summation index $p$ with $i$ and $j$, respectively. Then from (3)-(5), the BER minimization modulation diversity scheme $\min _{\lambda_{1}, \lambda_{2}} P_{B E R}$ becomes

$$
\begin{aligned}
& \min _{\mathbf{x}} \sum_{p=0}^{Q-1} \sum_{i=0}^{Q-1} \sum_{j=0}^{Q-1} x_{p i j} \sum_{l=0}^{Q-1} \sum_{q=0}^{Q-1} \sum_{k=0}^{Q-1} c_{p, i, j, q, k, l} \times x_{q k l}, \\
& \text { s.t.x } \in \mathcal{P} \cap \mathcal{I} \cap \mathcal{J} .
\end{aligned}
$$

in which

$$
\begin{aligned}
c_{p, i, j, q, k, l} & =\frac{B[p, q]}{Q} P_{h_{0}, h_{1}, h_{2}, v_{1}, v_{2}}\{\vartheta(p, i, j, q, k, l)<0\}, \\
\vartheta & =\left|h_{1}\left(\lambda_{0}[i]-\lambda_{0}[k]\right)+h_{2}\left(\lambda_{0}[j]-\lambda_{0}[l]\right)+v_{2}\right|^{2} \\
& +\left|h_{0}\left(\lambda_{0}[p]-\lambda_{0}[q]\right)+v_{1}\right|^{2}-\left|v_{1}\right|^{2}-\left|v_{2}\right|^{2} .
\end{aligned}
$$

It can be noted from the expression in (8) that the key to finding $c_{p, i, j, q, k, l}$ lies in the evaluation of $P_{h_{0}, h_{1}, h_{2}, v_{1}, v_{2}}\{\vartheta(p, i, j, q, k, l)$. In simple terms, this is the cumulative distribution function (CDF) of the random variable $\vartheta(p, i, j, q, k, l)$ of (5). Let us define the moment generating function (MGF) as

$$
\Phi_{\vartheta}(\rho)=\mathbb{E}_{\vartheta}[\exp (-\rho \vartheta)]
$$

Under the well known Rician channel model, $h_{m} \sim \mathcal{C N}\left(\mu_{h_{m}}, \sigma_{h_{m}}^{2}\right), m=0,1,2$, we can extend the method proposed in [46,47] to compute $P_{h_{0}, h_{1}, h_{2}, v_{1}, v_{2}}\{\vartheta(p, i, j, q, k, l)<0\}$ :

$$
P_{h_{0}, h_{1}, h_{2}, v_{1}, v_{2}}\{\vartheta(p, i, j, q, k, l)<0\} \approx \frac{1}{2 v} \sum_{t=1}^{v} \Re\left\{\Phi_{\vartheta}\left(\xi+j \xi \tau_{t}\right)\right\}+\tau_{t} \Im\left\{\Phi_{\vartheta}\left(\xi+j \xi \tau_{t}\right)\right\}
$$

where

$$
\tau_{t}=\tan ((t-1 / 2) \pi / v),
$$

and $\Re\{\cdot\}$ and $\Im\{\cdot\}$ denote the real and imaginary parts, respectively. The parameter $\xi$ is selected to ensure convergence of the integration, and $\xi=1 / 4$ was suggested in [47]. The size $v$ of the expansion (11) needs to be large when $P_{h_{0}, h_{1}, h_{2}, v_{1}, v_{2}}\{\vartheta(p, i, j, q, k, l)<0\}$ is small in order to maintain an acceptable numerical accuracy. To compute $\Phi_{\vartheta}(\rho)$, let us denote Gaussian random vectors $\mathbf{z}_{1}=\left[h_{0}, v_{1}\right]^{T}, \mathbf{z}_{2}=\left[h_{1}, h_{2}, v_{2}\right]^{T}$, such that $\mathbf{z}_{m} \sim \mathcal{C N}\left(\mu_{m}, \mathbf{\Sigma}_{m}\right), m=1,2$, where 


$$
\begin{aligned}
& \mu_{1}=\left[\mu_{h_{0}}, 0\right]^{T}, \\
& \mu_{2}=\left[\mu_{h_{1}}, \mu_{h_{2}}, 0\right]^{T}, \\
& \boldsymbol{\Sigma}_{1}=\operatorname{diag}\left(\sigma_{h_{0}}^{2}, \sigma_{v}^{2}\right), \\
& \boldsymbol{\Sigma}_{2}=\operatorname{diag}\left(\sigma_{h_{1}}^{2}, \sigma_{h_{2}}^{2}, \sigma_{v}^{2}\right) .
\end{aligned}
$$

Then (5) can be rewritten as $\vartheta=\mathbf{z}_{1}^{H} \mathbf{A}_{1} \mathbf{z}_{1}+\mathbf{z}_{2}^{H} \mathbf{A}_{2} \mathbf{z}_{2}$, where

$$
\mathbf{A}_{1}=\left[\begin{array}{cc}
\left|e_{p q}\right|^{2} & e_{p q}^{*} \\
e_{p q} & 0
\end{array}\right], \mathbf{A}_{2}=\left[\begin{array}{ccc}
\left|e_{i k}\right|^{2} & e_{i k}^{*} e_{j l} & e_{i k}^{*} \\
e_{i k} e_{j l}^{*} & \left|e_{j l}\right|^{2} & e_{j l}^{*} \\
e_{i k} & e_{j l} & 0
\end{array}\right]
$$

in which the notation $e_{a b}$ is given as

$$
e_{a b}=\lambda_{0}[a]-\lambda_{0}[b]
$$

Thus, the MGF is given as

$$
\Phi_{\vartheta}(\rho)=\sum_{m=1,2} \frac{\exp \left(-\rho \mu_{m}^{H} \mathbf{A}_{m}\left(\mathbf{I}+\rho \boldsymbol{\Sigma}_{m} \mathbf{A}_{m}\right)^{-1} \mu_{m}\right)}{\operatorname{det}\left(\mathbf{I}+\rho \boldsymbol{\Sigma}_{m} \mathbf{A}_{m}\right)} .
$$

Note that for any instance of quadratic assignment problem a total number of $Q^{6}$ coefficients must be computed. Fortunately, the modulation diversity design is based on statistical CSI which does not require online computation of the $Q^{6}$ coefficients in real-time. In fact, the optimized modulation diversity can be precomputed and stored a priori in our network nodes. In our simulation, we implemented the above procedure for 16-QAM and 32-QAM within $\mathrm{C}++$ on a workstation with 48 cores and finished the computation in several days for the $Q=32$ case and a few hours for the 16-QAM case. For a larger constellation such as 64-QAM, however, the time and spacial complexity may still be impractical. We will address in future works, new means to reduce this complexity by imposing rules to restrict the remapping schemes.

Applying large and complex algorithms, such as branch-and-bound techniques, to solve a relatively modest constellation size of 16-QAM and 32-QAM, is not feasible. Additionally, doing so may lead to some symmetry issues, thus, rendering it impractical. Here, we aim to find a solution using the iterative search technique. This algorithm works as follows. It starts by random mapping of $\lambda_{1}^{(0)}, \lambda_{2}^{(0)}$. It then executes a local search process, and whenever the objective function is reduced, the changing of mapping of indices is performed. This process continues to lower the objective and the local mapping is updated for each reduction. When a local minimum approaches, the algorithm executes a perturbation step by changing the mapping of $k_{p}$ indices. Note that $k_{p}$ indices are adaptively changed from $k_{p, \min }$ to $k_{p, \max }$. After the perturbation, the local search is again restarted until a pre-specified stopping criterion is met.

\subsection{A Heuristic Modulation Diversity Scheme}

For Rician fading channels, there is a strong LOS component in $h_{1}$ and $h_{2}$. Our quadratic assignment problem's solution benefits from two different gains. Firstly, by allowing $\lambda_{1} \neq \lambda_{0}$ and $\lambda_{2} \neq$ $\lambda_{0}$, we achieve the signal space diversity gain just like existing modulation diversity schemes. Secondly, by jointly designing $\lambda_{1}$ and $\lambda_{2}$ and allowing $\lambda_{1} \neq \lambda_{2}$, we achieve the cooperative gain between the source and the relay. When the channels are independently Rayleigh, however, there is little cooperative gain to exploit and we can greatly simplify the modulation diversity design problem by 
forcing equal HARQ mapping; i.e., $\lambda_{1}=\lambda_{2}$. Though we can solve the resulting quadratic assignment problem rigorously, from (5), we can take a simple heuristic approach noticing that the two indices mapped to two symbols close to each other in Phase 1 should be mapped to two symbols far apart in Phase 2. Based on such heuristic, $\lambda_{1}=\lambda_{2}$ can be designed for 16-QAM and 64-QAM. For 32-QAM constellation, a similar heuristic modulation diversity design may be extended by remapping the three most significant bits (MSBs) and the two least significant bits (LSBs) separately. We will show in the next section that, when channels experience deep fading, such a heuristic remapping method offers a comparable performance gain to that of our quadratic assignment problem-based modulation diversity.

\section{Numerical Results}

In our simulation, all Rician fading channels are assumed to have the same Rician parameter $K$. Additionally, we assume that during the second phase, the phases of the LOS components of channels $h_{1}$ and $h_{2}$ can be aligned at the source and relay, respectively. Consequently, we define $\mu_{h_{0}}=\mu_{h_{1}}=\sqrt{K /(K+1)}, \mu_{h_{2}}=a \sqrt{K /(K+1)}, \sigma_{h_{0}}^{2}=\sigma_{h_{1}}^{2}=1 /(K+1)$ and $\sigma_{h_{2}}^{2}=|a|^{2} /(K+1)$, where $a$ denotes the ratio between the amplitude of the LOS component of the relay to destination and the source to destination link. The noise power is parameterized with $E_{b} / N_{0}$ of the source to destination link.

First, we provide an example of quadratic assignment problem-optimized modulation diversity for 16-QAM. For $E_{b} / N_{0}=2 \mathrm{~dB}, K=10$ and $a=1$, the remapping schemes $\lambda_{1}$ and $\lambda_{2}$ are depicted in Figure 2. Here, "S-HARQ" denotes the simple HARQ retransmission, where $\lambda_{1}=\lambda_{2}=\lambda_{0}$, "Heuristic" indicates the conventional heuristic approach where $\lambda_{1}=\lambda_{2}=\lambda_{S}$ and "Proposed" represents the proposed modulation diversity solution. The results support the use of heuristics discussed in Section 3.2 as both $\lambda_{1}$ and $\lambda_{2}$ are essentially very close to $\lambda_{S}$. However, we note that in general $\lambda_{1} \neq \lambda_{2}$.

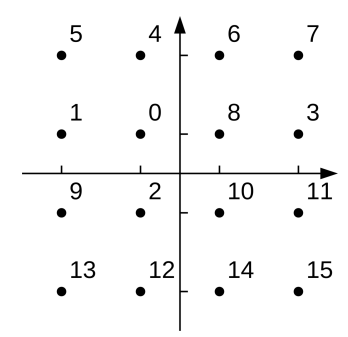

(a) $\lambda_{1}$

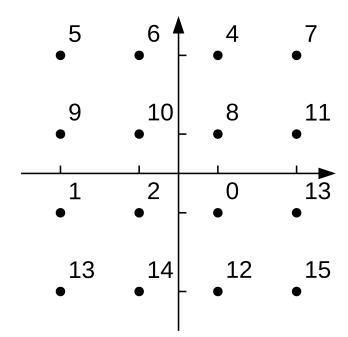

(b) $\lambda_{2}$

Figure 2. Illustration of constellations $\lambda_{1}$ and $\lambda_{2}$.

Next, we compare the empirical BER for three mapping schemes. The empirical BER is evaluated by applying the ML demodulator in (2) and over $M=10^{7}$ randomly generated channels, noise instances and data symbols. In Figure 3, there is a considerable performance gain for the proposed modulation diversity scheme versus S-HARQ. Moreover, the gain of the proposed scheme over $\lambda_{1}=\lambda_{2}=\lambda_{S}$ indicates that different rearrangements at the source and relay during the cooperative transmission can further reduce BER and boost performance. However, as $K$ decreases, heuristic modulation diversity becomes a good approximate solution with a diminishing performance gap from the proposed scheme.

Finally, we test the coded BER performance and the robustness of the modulation diversity design with a typical LDPC coded system. Although our proposed scheme depends on noise power and statistical CSI, in this simulation we only use modulation diversity schemes designed for the specific channel condition of $E_{b} / N_{0}=-2 d B$ under the additive white Gaussian noise (AWGN) channel. We test the modulation diversity design on Rician channels of $K=10$ and $a=1$, and various $E_{b} / N_{0}$ to demonstrate its robustness. The coded BER results for 16-QAM and 32-QAM are plotted in Figure 4a,b, respectively. We can see that the proposed solution provides additional gains 
over the heuristic approach and S-HARQ, even for mismatched design conditions. Additionally, we notice that the proposed solution provides a larger performance gain for 32-QAM than for 16-QAM constellation.

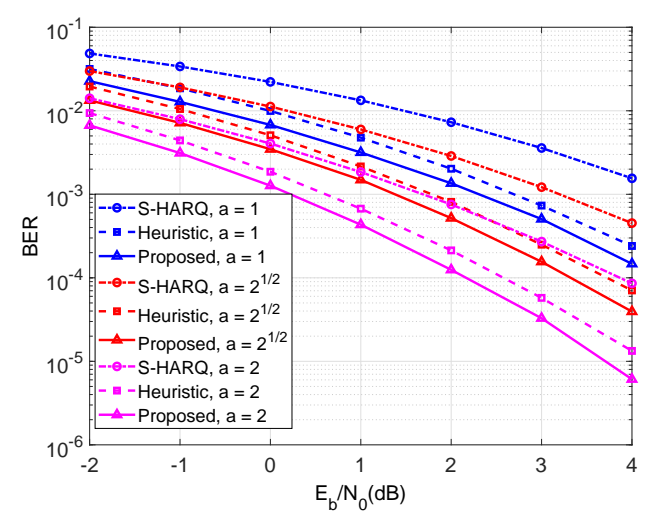

(a)

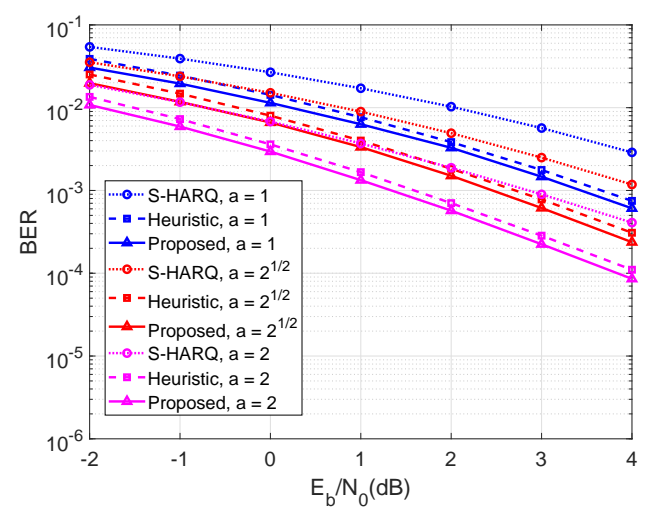

(b)

Figure 3. Monte-Carlo simulated BER of (1) quadratic assignment problem-optimized modulation diversity (2) $\lambda_{1}=\lambda_{2}=\lambda_{S}$ (3) $\lambda_{1}=\lambda_{2}=\lambda_{0}$ for $K=5,10, a=1, \sqrt{2}$, 2. Here, (a) $K=10$ (b) $K=5$.

Figure 5 shows the performance of the proposed technique against conventional S-HARQ and heuristic approaches. It can be noted that the proposed approach outperforms the other approaches. Note that "S-HARQ" here represents the simple HARQ retransmission, "Heuristic" indicates the conventional heuristic approach where $\lambda_{1}=\lambda_{2}=\lambda_{S}$, "Proposed (Bound)" indicates the upper performance bound of the proposed solution and "Proposed" represents the exact proposed modulation diversity solution. Note that the proposed solution is upper bounded by the dashed curve and the distance between the two curves reduces for higher values of signal power. Furthermore, for smaller values of signal power, the S-HARQ approach performs better, and at larger values the proposed bounded technique appears more suitable. 


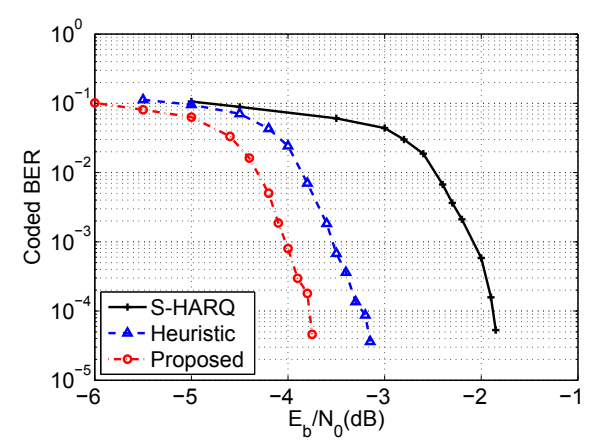

(a)

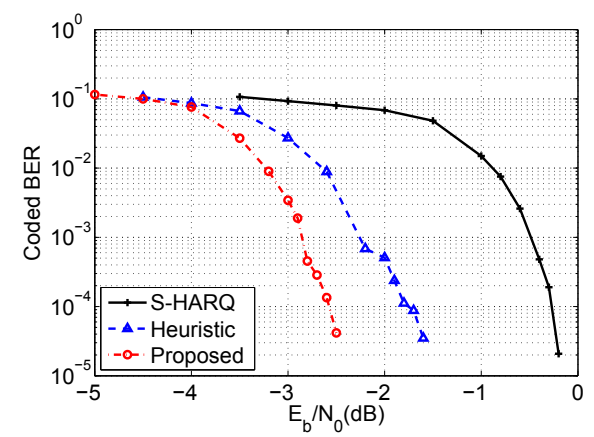

(b)

Figure 4. Monte-Carlo simulated coded BER with (a) 16-QAM, (b) 32-QAM.

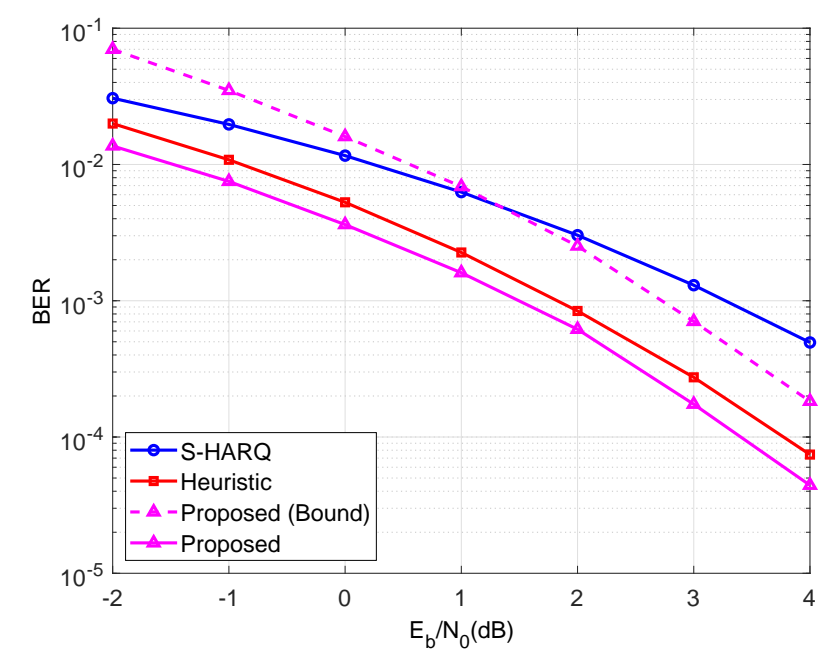

Figure 5. BER comparison of different schemes.

To further elaborate the significance of the proposed solution against different values of $K$, Figure 6 plots the BER when $K=5$ and $K=10$. It can be seen that different values of $K$ and $a$ have different impacts on the performance of the network. Note that at very small values of signal power, the BER for $K=10$ is better than $K=5$. However, it changes at very large values of signal power when the BER of $K=5$ is less than the BER of $K=10$. It is also worth pointing out that the difference between curves increases for larger signal power. 


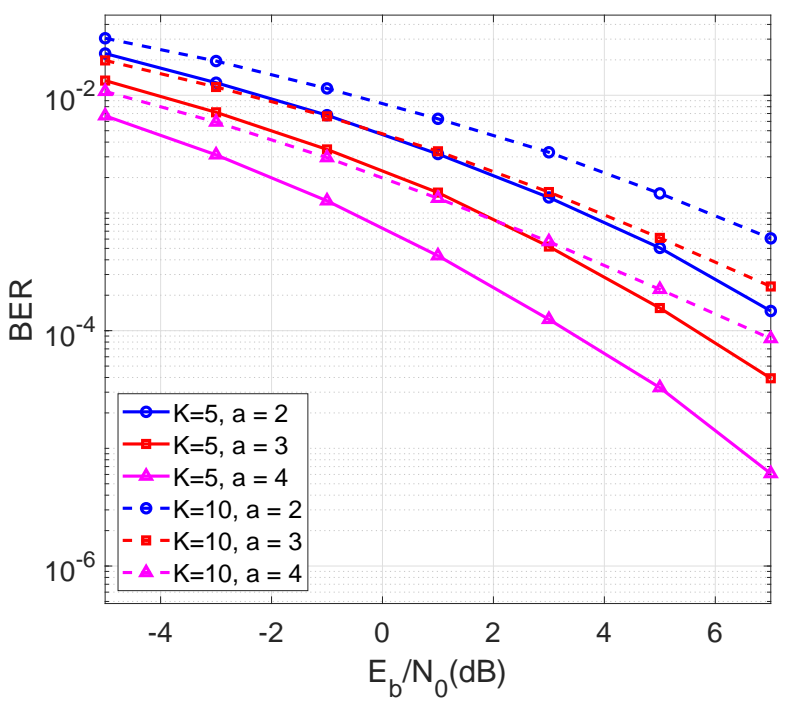

Figure 6. BER of proposed scheme for different values of $K$.

\section{Conclusions}

Efficient and reliable relay-aided IoT networks are going to play a pivotal in the widespread connectivity of low-powered devices. Using the coordinated retransmission from both the source and the relay, this work has provided an efficient modulation mapping solution for a relay-aided HARQ system. The modulation diversity design has been formulated as a quadratic assignment problem which is solved with the help of iterative search approach. The simulation results demonstrate the superiority of the proposed solution over simply repeated use of Gray mapping. The proposed approach also shows significant improvement when compared to the heuristic method.

Although the proposed solution significantly improves the performance of the network, there are many ways that it can be further improved. For instance, the impact of the proposed solution on multi-user interference-limited networks should be evaluated. Furthermore, the impact of imperfect channel information should also be explored. From the perspective of security, the effect of untrusted relays must be evaluated on the broadcast nature of wireless signals. These challenging but interesting tasks will be undertaken in future studies.

Author Contributions: Conceptualization: S.L.M.; methodology: I.K.; data curation: W.S.; formal analysis: A.B.F.; software: P.U.; writing —original draft: I.K.; funding acquisition: P.U.; supervision: S.L.M.; project administration: P.U.; writing-review and editing: S.B. and A.A.I.; visualization: A.A.I.; validation: S.B.; resources: A.A.I.; software: S.B. All authors have read and agreed to the published version of the manuscript.

Funding: This research is supported by the SUT Research and Development Fund.

Acknowledgments: The authors would like to say thanks to the reviewers and editors for reviewing this manuscript.

Conflicts of Interest: The authors declare no conflict of interest.

\section{Abbreviations}

The following abbreviations are used in this manuscript: 


$\begin{array}{ll}\text { DF } & \text { Decode-and-forward } \\ \text { IoT } & \text { Internet-of-Things } \\ \text { UAV } & \text { Unmanned aerial vehicle } \\ \text { BER } & \text { Bit error rate } \\ \text { HARQ } & \text { Hybrid automatic repeat request } \\ \text { MISO } & \text { Multiple-input single-output } \\ \text { CSI } & \text { Channel state information } \\ \text { ML } & \text { Maximum likelihood } \\ \text { PEP } & \text { Pair-wise error probability } \\ \text { CDF } & \text { Cumulative distribution function } \\ \text { MGF } & \text { Moment generating function } \\ \text { LOS } & \text { Line-of-sight } \\ \text { MBS } & \text { Most significant bits } \\ \text { LBS } & \text { Least significant bits }\end{array}$

\section{References}

1. Wen, M.; Zheng, B.; Kim, K.J.; Di Renzo, M.; Tsiftsis, T.A.; Chen, K.C.; Al-Dhahir, N. A survey on spatial modulation in emerging wireless systems: Research progresses and applications. IEEE J. Sel. Areas Commun. 2019, 37, 1949-1972. [CrossRef]

2. Jameel, F.; Duan, R.; Chang, Z.; Liljemark, A.; Ristaniemi, T.; Jantti, R. Applications of Backscatter Communications for Healthcare Networks. IEEE Netw. 2019, 33, 50-57. [CrossRef]

3. Shahjehan, W.; Riaz, A.; Khan, I.; Sadiq, A.S.; Khan, S.; Khan, M.K. Bat algorithm-based beamforming for mmWave massive MIMO systems. Int. J. Commun. Syst. 2020, 33, e4182. [CrossRef]

4. Shahjehan, W.; Shah, S.W.; Lloret, J.; Bosch, I. A Novel Codeword Selection Scheme for MIMO-MAC Lower-Bound Maximization. Entropy 2018, 20, 546. [CrossRef]

5. Jameel, F.; Ristaniemi, T.; Khan, I.; Lee, B.M. Simultaneous harvest-and-transmit ambient backscatter communications under Rayleigh fading. EURASIP J. Wirel. Commun. Netw. 2019, 2019, 166. [CrossRef]

6. Awais, M.; Raza, M.; Ali, K.; Ali, Z.; Irfan, M.; Chughtai, O.; Khan, I.; Kim, S.; Ur Rehman, M. An Internet of Things based bed-egress alerting paradigm using wearable sensors in elderly care environment. Sensors 2019, 19, 2498. [CrossRef] [PubMed]

7. Saraereh, O.A.; Ali, A.; Khan, I.; Rabie, K. Interference Analysis for Vehicle-to-Vehicle Communications at 28 GHz. Electronics 2020, 9, 262. [CrossRef]

8. Saraereh, O.A.; Alsaraira, A.; Khan, I.; Choi, B.J. A Hybrid Energy Harvesting Design for On-Body Internet-of-Things (IoT) Networks. Sensors 2020, 20, 407. [CrossRef]

9. Ma, D.; Lan, G.; Hassan, M.; Hu, W.; Das, S.K. Sensing, Computing, and Communications for Energy Harvesting IoTs: A Survey. IEEE Commun. Surv. Tutor. 2019. [CrossRef]

10. Chakareski, J. UAV-IoT for next generation virtual reality. IEEE Trans. Image Process. 2019, 28, 5977-5990. [CrossRef]

11. Saraereh, O.A.; Khan, I.; Lee, B.M. An efficient neighbor discovery scheme for mobile WSN. IEEE Access 2018, 7, 4843-4855. [CrossRef]

12. Saraereh, O.A.; Alsaraira, A.; Khan, I.; Uthansakul, P. An Efficient Resource Allocation Algorithm for OFDM-Based NOMA in 5G Systems. Electronics 2019, 8, 1399. [CrossRef]

13. Jameel, F.; Wyne, S.; Krikidis, I. Secrecy outage for wireless sensor networks. IEEE Commun. Lett. 2017, 21, 1565-1568. [CrossRef]

14. Bakht, K.; Jameel, F.; Ali, Z.; Khan, W.U.; Khan, I.; Sidhu, S.; Ahmad, G.; Lee, J.W. Power Allocation and User Assignment Scheme for beyond 5G Heterogeneous Networks. Wirel. Commun. Mob. Comput. 2019, 2019, 2472783. [CrossRef]

15. 3GPP TS36.331. E-UTRA Radio Resource Control (RRC); Protocol Specification (Release 12) 2015. v12.5.0. Available online: https:/ / www.etsi.org/deliver/etsi_ts/136300_136399/136331/12.05.00_60/ts_ 136331v120500p.pdf (accessed on 1 March 2020).

16. Ngo, H.A.; Hanzo, L. Hybrid Automatic-Repeat-reQuest Systems for Cooperative Wireless Communications. IEEE Commun. Surv. Tutor. 2014, 16, 25-45. [CrossRef] 
17. Sahin, C.; Liu, L.; Perrins, E.; Ma, L. Delay-sensitive communications over IR-HARQ: Modulation, coding latency, and reliability. IEEE J. Sel. Areas Commun. 2019, 37, 749-764. [CrossRef]

18. Seddik, K.; Ibrahim, A.; Liu, K. Trans-Modulation in Wireless Relay Networks. IEEE Commun. Lett. 2008, 12, 170-172. [CrossRef]

19. Jameel, F.; Wyne, S.; Kaddoum, G.; Duong, T.Q. A comprehensive survey on cooperative relaying and jamming strategies for physical layer security. IEEE Commun. Surv. Tutor. 2018, 21, 2734-2771. [CrossRef]

20. Ryu, H.S.; Lee, J.S.; Kang, C. BER Analysis of Constellation Rearrangement for Cooperative Relaying Networks over Nakagami- $m$ Fading Channel. In Proceedings of the 2011 IEEE International Conference on Communications (ICC), Kyoto, Japan, 5-9 June 2011. [CrossRef]

21. Bedoui, A.; Et-tolba, M.; Nouasria, H. An MMSE Integrated Equalization for HARQ Chase Combining in OQAM-FBMC systems. In Proceedings of the IEEE 2019 15th International Wireless Communications \& Mobile Computing Conference (IWCMC), Tangier, Morocco, 24-28 June 2019; pp. 811-816.

22. Leturc, X.; Ciblat, P.; Le Martret, C.J. Energy efficient resource allocation for Type-I HARQ under the Rician channel. IEEE Trans. Wirel. Commun. 2019, 18, 3739-3751. [CrossRef]

23. Xiang, Z.; Yang, W.; Pan, G.; Cai, Y.; Song, Y.; Zou, Y. Secure Transmission in HARQ-Assisted Non-orthogonal Multiple Access Networks. IEEE Trans. Inf. Forensics Secur. 2019, 15, 2171-2182. [CrossRef]

24. Xia, H.; Han, S.; Li, C.; Meng, W. Joint PHY / MAC Layer AN-Assisted Security Scheme in SVD-Based MIMO HARQ system. In Proceedings of the 2019 IEEE/CIC International Conference on Communications in China (ICCC), Changchun, China, 11-13 August 2019; pp. 328-333.

25. Baldi, M.; Maturo, N.; Ricciutelli, G.; Chiaraluce, F. Physical layer security over fading wiretap channels through classic coded transmissions with finite block length and discrete modulation. Phys. Commun. 2019, 37, 100829. [CrossRef]

26. Chen, K.; Niu, K.; Lin, J. A hybrid ARQ scheme based on polar codes. IEEE Commun. Lett. 2013, 17, $1996-1999$. [CrossRef]

27. Saber, H.; Marsland, I. An incremental redundancy hybrid ARQ scheme via puncturing and extending of polar codes. IEEE Trans. Commun. 2015, 63, 3964-3973. [CrossRef]

28. Liang, H.; Liu, A.; Zhang, Y.; Liang, X. Efficient design of multi-packet hybrid ARQ transmission scheme based on polar codes. IEEE Access 2018, 6, 31564-31570. [CrossRef]

29. Eslami, A.; Pishro-Nik, H. A practical approach to polar codes. In Proceedings of the 2011 IEEE International Symposium on Information Theory Proceedings, St. Petersburg, Russia, 31 July-5 August 2011; pp. 16-20.

30. Niu, K.; Chen, K.; Lin, J.R. Beyond turbo codes: Rate-compatible punctured polar codes. In Proceedings of the 2013 IEEE International Conference on Communications (ICC), Budapest, Hungary, 9-13 June 2013; pp. 3423-3427.

31. Zhao, M.M.; Zhang, G.; Xu, C.; Zhang, H.; Li, R.; Wang, J. An adaptive IR-HARQ scheme for polar codes by polarizing matrix extension. IEEE Commun. Lett. 2018, 22, 1306-1309. [CrossRef]

32. Liang, H.; Liu, A.; Zhang, Y.; Cheng, F.; Yi, X. A Throughput-Efficient Rateless Scheme of Polar Codes. In Proceedings of the IEEE 2018 10th International Conference on Wireless Communications and Signal Processing (WCSP), Hangzhou, China, 18-20 October 2018; pp. 1-7.

33. Gao, J.; Fan, P.; Li, L. Optimized Polarizing Matrix Extension based HARQ Scheme for Short Packet Transmission. IEEE Commun. Lett. 2020. [CrossRef]

34. Song, E.C.; Yue, G. Finite Blocklength Analysis for Coded Modulation with Applications to Link Adaptation. In Proceedings of the 2019 IEEE Wireless Communications and Networking Conference (WCNC), Marrakesh, Morocco, 15-18 April 2019; pp. 1-7.

35. Wang, Z.; Dang, S.; Kennedy, D.T. Multi-hop index modulation-aided OFDM with decode-and-forward relaying. IEEE Access 2018, 6, 26457-26468. [CrossRef]

36. Zhao, Y.; Hu, J.; Ding, Z.; Yang, K. Joint interleaver and modulation design for multi-user SWIPT-NOMA. IEEE Trans. Commun. 2019, 67, 7288-7301. [CrossRef]

37. Pechetti, S.V.; Bose, R. Precoding-aided Spatial Modulation Assisted Joint Two-Tier Downlink Reception. IEEE Trans. Wirel. Commun. 2020. [CrossRef]

38. Jiang, L.; Li, X.; Ye, N.; Wang, A. Deep Learning-Aided Constellation Design for Downlink NOMA. In Proceedings of the IEEE 2019 15th International Wireless Communications \& Mobile Computing Conference (IWCMC), Tangier, Morocco, 24-28 June 2019; pp. 1879-1883. 
39. Saeed Khan, A.; Chatzigeorgiou, I.; Zheng, G.; Basulti, B.; Chuma, J.; Lambotharan, S. Random linear network coding based physical layer security for relay-aided device-to-device communication. IET Commun. 2020, 17, 33.

40. Wu, W.; Mittelmann, H.; Ding, Z. Modulation design for two-way amplify-and-forward relay HARQ. IEEE Wirel. Commun. Lett. 2016, 5, 244-247. [CrossRef]

41. Benlic, U.; Hao, J.K. Memetic search for the quadratic assignment problem. Expert Syst. Appl. 2015, 42, 584-595. [CrossRef]

42. Hu, J.; Zhao, Y.; Yang, K. Modulation and coding design for simultaneous wireless information and power transfer. IEEE Commun. Mag. 2019, 57, 124-130. [CrossRef]

43. Zheng, K.; Hu, L.; Wang, W.; Huang, L. Performance analysis of HARQ transmission in cooperative DF relaying systems. Wirel. Pers. Commun. 2010, 55, 441-455. [CrossRef]

44. Ozkan, I. Analysis of Cooperative Hybrid ARQ with Adaptive Modulation and Coding on a Correlated Fading Channel. arXiv 2019, arXiv:1910.07959.

45. Kim, J.W.; Lee, H.; Ahn, J.; Kang, C. Design of Signal Constellation Rearrangement (CoRe) for Multiple Relay Links. In Proceedings of the GLOBECOM 2009-2009 IEEE Global Telecommunications Conference, Honolulu, HI, USA, 30 November-4 December 2009; pp. 1-6. [CrossRef]

46. Samra, H.; Ding, Z.; Hahn, P. Symbol mapping diversity design for multiple packet transmissions. IEEE Trans. Commun. 2005, 53, 810-817. [CrossRef]

47. Taricco, G.; Biglieri, E. Exact pairwise error probability of space-time codes. EEE Tran. Inf. Theory 2002, 48, 510-513. [CrossRef]

(C) 2020 by the authors. Licensee MDPI, Basel, Switzerland. This article is an open access article distributed under the terms and conditions of the Creative Commons Attribution (CC BY) license (http:/ / creativecommons.org/licenses/by/4.0/). 